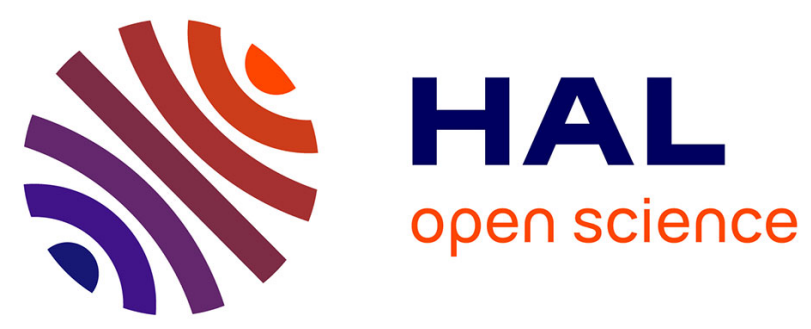

\title{
TVA et taux de marge: une analyse empirique sur données d'entreprises exportatrices françaises
}

Philippe Andrade, Martine Carré, Agnès Bénassy-Quéré

\section{To cite this version:}

Philippe Andrade, Martine Carré, Agnès Bénassy-Quéré. TVA et taux de marge: une analyse empirique sur données d'entreprises exportatrices françaises. Economie \& prévision, 2012, 2-3 (200-201), pp.22. hal-01515876

\author{
HAL Id: hal-01515876 \\ https://hal.science/hal-01515876
}

Submitted on 28 Apr 2017

HAL is a multi-disciplinary open access archive for the deposit and dissemination of scientific research documents, whether they are published or not. The documents may come from teaching and research institutions in France or abroad, or from public or private research centers.
L'archive ouverte pluridisciplinaire HAL, est destinée au dépôt et à la diffusion de documents scientifiques de niveau recherche, publiés ou non, émanant des établissements d'enseignement et de recherche français ou étrangers, des laboratoires publics ou privés. 
No $2010-30$

Décembre

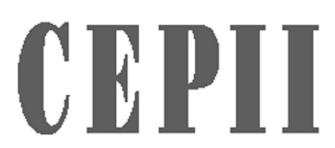

CENTRE

D'ÉTUDES PROSPECTIVES

ET D'INFORMATIONS

INTERNATIONALES

TVA et taux de marge :

Une analyse empirique sur données d'entreprises

Philippe Andrade, Martine Carré et Agnès Bénassy-Quéré 


\section{TABLE DES MATIÈRES}

Non-technical summary. . . . . . . . . . . . . . . . . . . . . . . . . . . 3

Abstract . . . . . . . . . . . . . . . . . . . . . . . . . . . . . . 4

Résumé non technique . . . . . . . . . . . . . . . . . . . . . . 5

Résumé court . . . . . . . . . . . . . . . . . . . . . . . . . . . . 6

1. Introduction . . . . . . . . . . . . . . . . . . . . . . . . . . . . . . 7

2. Un modèle empirique de concurrence imparfaite sur les marchés internationaux . . . 9

2.1. Taux de marge optimal des exportateurs . . . . . . . . . . . . . . . . . 9

2.2. Choc de TVA et variations optimales des taux de marge . . . . . . . . . . . 10

2.3. Stratégie Économétrique . . . . . . . . . . . . . . . . . . . . . . . 11

3. Données . . . . . . . . . . . . . . . . . . . . . . . . 13

4. Resultats . . . . . . . . . . . . . . . . . . . . . . . 14

5. Conclusion . . . . . . . . . . . . . . . . . . . . . . . . . . . . . 16 


\section{VAT SHOCKS AND MARK-UPS: AN EMPIRICAl ANALYSis BASEd ON Firm-LEVEL DATA}

\section{NON-TECHNICAL SUMMARY}

Raised on the destination principle, the Valued-Added Tax (VAT) is often considered one of the rare levies that have little spillover on foreign economies. It is also neutral in the sense that it affects consumption prices of all goods in a similar way, be they produced locally or imported: VAT does not directly distort relative prices, hence the optimal choices of the consumers and the producers.

This international neutrality does not mean that producers do not react to changes in VAT rates, neither that their reaction is uniform across firms and sectors: to the extent that a higher consumption price reduces the demand for the corresponding goods and services, it is optimal for producers to reduce their margins, in variable proportions depending on the price-elasticity of the demand and on firms' profitability. Hence, a variation in VAT rates can have redistributive effects across sectors and firms, not only in the country where the policy is applied, but also in foreign supplier countries.

We study the way exporters from one country (here, France) react to a VAT shock in a destination country, based on French customs data that detail both volumes and values of exported goods at the firm level, over the 1995-2005 period, for around 10,000 products (NC8 classification). The use of individual data allows us to evidence some heterogeneity of firms reaction depending, in particular, on their market shares.

Besides its implications for tax policies, our work sheds some new light on the more general question of imperfect transmission of macroeconomic shocks to prices in an open economy. To date, this question has essentially been scrutinized by studying exchange-rate pass-through. In an authoritative literature survey, Goldberg \& Knetter (1997) conclude that exchange-rate pass-through is around 50\% after one year: when the nominal exchange rate of a country appreciates by $10 \%$, its exporters' prices on foreign markets increase by $5 \%$ on average after one year, the remaining being absorbed through compressed margins.

Our contribution to this literature is to measure the extent of pass-through after a VAT rather than an exchange-rate shock. Focusing on VAT has three main advantages. Firstly, contrasting with the exchange rate, VAT has no direct impact on the marginal cost of producers. Therefore, any producer-price adjustment can be attributed to mark-up changes. Secondly, changes in VAT rates are the results of policy decisions that by nature are unfrequent and exogenous. Hence, VAT changes can be expected to be long-lasting. This contrasts with exchange-rate volatility that can trigger less reaction from the producers, who will expect the shocks to be reversed. Thirdly, VAT applies in a symmetric way to imported and locally-produced goods. Thus, the reaction of producer prices cannot be attributed to changes in relative prices following the shock.

Our results suggest an average pass-through of $48 \%$ for final consumption goods: when the VAT rate of a destination country rises by $10 \%$ (for instance, from 20 to $22 \%$ ), French exporters increase their price at 
the consumer's level by an average 4,8\% (hence, they reduce their before-tax prices by an average 5,2\%). This pass-through figure is higher than recent empirical estimates based on individual data for the case of exchange-rate shocks (see, for instance, Berman et al. (2009) or Gopinath \& Rigobon (2008) who find an average pass-through of no more than 20\%). Following Berman et al. (2009), we further evidence a composition effect: the VAT shock changes the population of exporters, so the aggregate coefficient tends to represent the behavior of surviving firms, which are those that are more able to absorb the shock in their margins. Beyond this result, we show that the firms that absorb the shock the most in their margins are those displaying either very high or very low market shares in the destination market. Only a detailed classification of the firms depending on their market shares allows us to evidence such non-linear effect.

The highly incomplete pass-through observed after a symmetric, permanent shock (a VAT shock) tends to validate theoretical models that do not rely on a constant price-elasticity of demand. On the policy side, our results suggest that consumption prices will not react one-for-one after a VAT hike, but that markup compression will affect market structures. Our results also suggest that changes in VAT rates in country do have implications beyond national frontiers.

\begin{abstract}
We study how French exporters react to a VAT shock in a destination country. VAT shocks are by nature almost permanent, exogenous, and have no impact on marginal costs. We argue that the subsequent price reaction therefore identifies to which extent mark-up adjustments tame the impact of a macro shock on final consumption prices. We show that even a uniform increase in the VAT rate can entail non-uniform mark-up adjustments across exporters and, consequently, an important redistribution of profits both between sectors and across firms within a sector. Indeed, mark-up adjustments vary with the elasticity of demand and the market shares.
\end{abstract}

JEL Classification: $\mathrm{H} 21, \mathrm{H} 23, \mathrm{~J} 41$

Keywords: $\quad$ Value-Added Tax (VAT), Pass-through, Firm data, Export prices. 
TVA ET TAUX DE MARGE : UNE ANALYSE EMPIRIQUE SUR DONNÉES D'ENTREPRISES

\section{RÉSUME NON TECHNIQUE}

Impôt prélevé selon le principe de la destination, la Taxe sur la Valeur Ajoutée (TVA) est souvent considérée comme l'un des rares prélèvements dont les effets restent cantonnés au seul territoire national. C'est aussi un impôt neutre au sens où il frappe de la même manière les prix à la consommation de tous les biens et services, qu'ils soient produits localement ou importés : la TVA ne distord pas directement les prix relatifs et donc les choix des agents en matière de consommation et de production.

Cette neutralité internationale ne signifie pas que les producteurs ne réagissent pas à une variation de taux de TVA, ni que cette réaction est uniforme selon les secteurs et selon les firmes : dans la mesure où un renchérissement des biens et services réduit le volume de consommation, il est optimal pour les producteurs de comprimer leur taux de marge, dans une proportion variable selon l'élasticité-prix de la demande et la profitabilité des entreprises. Ainsi, une variation de taux de TVA peut avoir des effets redistributifs entre secteurs et entre les firmes d'un même secteur, non seulement dans le pays où a lieu le choc de TVA, mais aussi au sein des pays fournisseurs étrangers.

Nous étudions la manière dont les exportateurs d'un pays (dans notre cas, la France) réagissent à un choc de TVA dans un pays de destination. Nous exploitons pour cela une base de données des douanes françaises retraçant les volumes et les valeurs des marchandises exportées au niveau de chaque entreprise au cours de la période 1995-2005, au niveau NC8 (soit environ 10000 produits). L'utilisation de données individuelles permet de mettre en évidence une réaction hétérogène des entreprises selon, en particulier, leurs parts de marché.

Outre les enseignements que l'on peut tirer pour la politique fiscale, ce travail apporte un nouvel éclairage sur la qustion plus générale de la transmission imparfaite des chocs macroéconomiques aux prix dans une économie ouverte, question jusqu'ici essentiellement traitée sous l'angle des répercussions des chocs de taux de change. Dans une revue de litérature faisant référence, Goldberg \& Knetter (1997) font état d'un "pass-through" de 50\% un an après un choc de taux de change : lorsque le taux de change nominal d'un pays s'apprécie de $10 \%$, les prix des exportateurs sur les marchés étrangers augmentent en moyenne de $5 \%$ un an après le choc, le reste étant absorbé par une compression des marges.

Nous renouvelons ici la littérature sur le sujet en nous intéresant non pas à un choc de taux de change, mais à un choc de TVA. Ceci présente trois principaux avantages. Premièrement, contrairement au taux de change, la TVA est sans effet direct sur le coût marginal des producteurs. L'ajustement des prix de production peut donc être attribué sans équivoque à une modification du taux de marge des entreprises. Deuxièmement, les variations des taux de TVA résultent de décisions de politique économique par nature peu fréquents et exogènes. On s'attend généralement à ce que le taux de TVA demeure pour plusieurs années au niveau décidé par les autorités, contrairement au taux change dont la forte volatilité fait qu'il 
peut être optimal de ne pas réagir à une variation qui peut facilement être jugée transitoire. Troisièmement, la TVA s'applique d'une façon symétrique aux marchandises importées et produites localement. Ainsi, l'ajustement des prix à la production ne peut être attribué à aucune altération des prix relatifs internationaux à la suite du choc.

Les résultats suggèrent un pass-through moyen de $48 \%$ pour les biens de consommation finale : lorsque TVA augmente de 10\% dans un pays de destination (par exemple, de 20 à 22\%), les exportateurs français relèvent en moyenne de 4,8\% leurs prix au niveau du consommateur final (ils réduisent donc de 5,2\% leurs prix avant impôt). Ce chiffre est plus élevé que ceux obtenus récemment dans différentes études empiriques sur données individuelles pour le cas du taux de change (voir, par exemple, Berman et al. (2009) ou Gopinath \& Rigobon (2008) qui obtiennent des coefficients moyens de pass-through ne dépassant pas 20\%. A la suite de Berman et al. (2009), nous mettons en évidence l'existence d'un effet de composition dans ce résultat : le choc modifie la population des entreprises présentes sur le marché, de telle sorte que le coefficient agrégé retrace le comportement des entreprises qui survivent et qui ont tendance à absorber plus fortement le choc dans leur taux de marge. Au-delà de ce résultat, nous montrons que les entreprises disposant de parts de marché très élevées ou au contraire très faibles absorbent le choc de façon plus importante que les entreprises "moyennes". Seule une classification assez fine des entreprises selon leurs parts de marché permet de détecter cette non linéarité selon la part de marché.

Sur le plan théorique, la présence d'un pass-through largement incomplet dans le cas d'un choc symétrique et permanent (un choc de TVA) tend à valider les modèles théoriques dans lesquels l'élasticité de la demande n'est pas supposée constante. Sur le plan pratique, ce résultat signifie que les prix à la consommation ne réagiront pas "un pour un" à une hausse du taux de TVA, mais que la compression des marges des producteurs modifiera la structure concurrentielle des marchés en réduisant le nombre de fournisseurs. Il suggère aussi que les variations de taux de TVA ont des effets qui dépassent les frontières nationales.

\section{RÉSUMÉ COURT}

Nous étudions la manière dont les exportateurs d'un pays réagissent à un choc de TVA dans un pays de destination. Les chocs de TVA étant par nature quasi permanents, exogènes et sans impact sur le coût marginal, l'étude de leurs répercussions sur les prix permet d'identifier dans quelle mesure les taux de marge permettent d'amortir l'impact du choc sur les prix à la consommation. Nous montrons que même une hausse uniforme du taux de TVA peut induire une modification non-uniforme des taux de marge des exportateurs, et par conséquent des effets redistributifs importants entre les secteurs et entre les entreprises d'un même secteur. Le comportement de marge est affecté par les variations de l'élasticité de la demande adressée aux exportateurs, et dépend de la part de marché des entreprises.

Classification JEL : $\mathrm{H} 21, \mathrm{H} 23, \mathrm{~J} 41$

Mots clés : $\quad$ Taxe à la Valeur Ajoutée (TVA), Pass-through, Données de firmes, Prix à l'exportation. 
TVA ET TAUX DE MARGE : UNE ANALYSE EMPIRIQUE SUR DONNÉES D'ENTREPRISES ${ }^{1}$

\author{
Philippe Andrade*, Martine Carré ${ }^{\dagger}$ and Agnès Bénassy-Quéré ${ }^{\ddagger}$
}

\title{
1. INTRODUCTION
}

Impôt prélevé selon le principe de la destination, la Taxe sur la Valeur Ajoutée (TVA) est souvent considérée comme l'un des rares prélèvements dont les effets restent cantonnés au seul territoire national. C'est aussi un impôt neutre au sens où il frappe de la même manière les prix à la consommation de tous les biens et services, qu'ils soient produits localement ou importés. Ainsi, même lorsque le taux de TVA ne varie que dans un seul pays, le choc fiscal est parfaitement symétrique pour l'ensemble des producteurs quelle que soit leur nationalité : en augmentant le prix de la quasi-totalité des biens dans une même proportion, une hausse de TVA ne distord pas directement les prix relatifs et donc les choix des agents en matière de consommation et de production.

Cette neutralité internationale ne signifie pas que les producteurs ne réagissent pas à une variation de taux de TVA, ni que cette réaction est uniforme selon les secteurs et selon les firmes : dans la mesure où un renchérissement des biens et services réduit le volume de consommation, il est optimal pour les producteurs de comprimer leur taux de marge, dans une proportion variable selon l'élasticité-prix de la demande et la profitabilité des entreprises. Ainsi, une variation de taux de TVA peut avoir des effets redistributifs entre secteurs et entre les firmes d'un même secteur, non seulement dans le pays où a lieu le choc de TVA, mais aussi au sein des pays fournisseurs étrangers.

Nous étudions la manière dont les exportateurs d'un pays (dans notre cas, la France) réagissent à un choc de TVA dans un pays de destination. S'il est optimal pour les producteurs de modifier leur taux de marge, la réforme de la fiscalité indirecte va modifier la répartition des profits au sein de chaque secteur car seules les entreprises les plus productives seront à même de diminuer leur taux de marge et d'absorber une partie de l'augmentation des prix pour préserver le niveau de la demande qui leur est adressée. De plus, l'augmentation du taux de TVA introduit des

\footnotetext{
${ }^{1}$ Nous tenons à remercier C. Carbonnier, M. Crozet, I. Méjean, M. Mélitz et C. Schwellnus, ainsi que les participants des séminaires du FMI, U-Cergy, UCLA, UCSD, U-Cyprus, et des Congrès de l'EEA 2009, de la SED, et de l'AFSE 2010. Cette contribution, qui a bénéficié du soutien de l'ANR dans le cadre du projet JCJC "Itace" (Indirect Tax and Competition in Europe), ne reflète pas nécessairement le point de vue de la Banque de France.

*Banque de France (philippe.andrade@ banque-france.fr)

${ }^{\dagger}$ LEDa-SDFi, Université de Paris-Dauphine (martine.carre-tallon@ dauphine.fr)

${ }_{\ddagger}^{\ddagger}$ CEPII (agnes.benassy@cepii.fr)
} 
distorsions entre les secteurs. Selon l'état de la concurrence et l'élasticité de la demande, le partage de l'augmentation du prix entre producteur et consommateur n'est pas identique. Une variation du taux de TVA modifie donc la distribution des taux de marge à l'intérieur et entre les secteurs d'activité. Or une concentration industrielle accrue dans un secteur pour lequel le taux de marge moyen est initialement supérieur au reste de l'économie amplifie les distorsions au sens de Lerner (1934) et les pertes en bien-être qui y sont associées.

Outre ses implications pour la politique fiscale, ce travail apporte un nouvel éclairage sur la question plus générale de la transmission imparfaite des chocs macroéconomiques aux prix dans une économie ouverte, question jusqu'ici traitée essentiellement sous l'angle des répercussions des chocs de taux de change. Cette littérature, initiée à la fin des années 1980 avec les apports notables de Knetter (1989), Feenstra (1989) et Knetter (1993), s'intéresse à la manière dont les entreprises absorbent le choc par un ajustement de leur taux de marge. Si les producteurs restaient passifs et ne modifiaient pas leur prix de production, le "pass-through" du choc au prix final serait complet. En d'autres termes, la modification du taux de change, ou pour notre étude, du taux de TVA, serait à $100 \%$ répercutée dans le prix au consommateur. A l'opposé, si les producteurs absorbaient l'intégralité du choc, laissant le prix à la consommation inchangé, le "pass-through" serait nul, égal à $0 \%$. Dans une revue de litérature faisant référence, Goldberg \& Knetter (1997) font état d'un "pass-through" de 50 \% un an après le choc. Goldberg \& Campa (2008), ainsi que Bouakez \& Rebei (2008) fournissent des preuves empiriques plus récentes de "pass through" incomplets, suggérant que les exportateurs lissent bien le choc en ajustant leur taux de marge.

Se concentrer sur un choc de TVA plutôt que sur une modification du taux de change présente trois principaux avantages. D'abord, un changement de taux de TVA dans un pays étranger affecte le prix à la consommation dans ce pays sans modifier le coût marginal des producteurs nationaux et étrangers. L'ajustement des prix de production peut alors être attribué sans équivoque à une modification du taux de marge des entreprises. Deuxièmement, les variations de taux de TVA résultent de décisions de politique économique par nature peu fréquents et exogènes. On s'attend généralement à ce que le taux de TVA demeure pendant plusieurs années au niveau décidé par les autorités, contrairement au taux de change dont la forte volatilité fait qu'il peut être optimal de ne pas réagir à une variation qui peut être jugée transitoire. Troisièmement, la TVA s'applique d'une façon symétrique aux marchandises importées et locales. Ainsi, l'ajustement des prix à la production ne peut être attribué à aucune altération des prix relatifs internationaux à la suite du choc.

Nous exploitons une base de données des douanes françaises retraçant les volumes et les valeurs des marchandises exportées au niveau de chaque entreprise au cours de la période 1995-2005, au niveau NC8 (soit environ 10000 produits). L'utilisation de données individuelles au niveau des entreprises nous permet de mettre en évidence l'hétérogénité des entreprises dans la réaction de prix aux modifications de taux de TVA.

Les résultats suggèrent un "pass-through" moyen de $48 \%$ pour les biens de consommation fi- 
nale. Ce chiffre est plus élevé que ceux obtenus récemment dans différentes études empiriques sur données individuelles pour le cas du taux de change ${ }^{2}$. A la suite de Berman et al. (2009), nous mettons en évidence l'existence d'un effet de composition dans ce résultat. Le choc modifie la population des entreprises présentes sur le marché de telle sorte que le coefficient agrégé retrace le comportement des entreprises qui survivent et qui ont tendance à absorber plus fortement le choc dans leur taux de marge. Au-delà de ce résultat, nous montrons que les entreprises disposant de parts de marché très fortes ou au contraire très faibles absorbent le choc de façon plus importante que les entreprises "moyennes". Seule une classification assez fine des entreprises selon leur niveau de part de marché permet de détecter cette non linéarité dans la réaction des entreprises.

\section{UN MODÈLE EMPIRIQUE DE CONCURRENCE IMPARFAITE SUR LES MARCHÉS IN- TERNATIONAUX}

\subsection{Taux de marge optimal des exportateurs}

Considérons une entreprise $f$ vendant un bien $k=1, \ldots, K$ sur différents marchés de destination $l=1, \ldots, L$. On note $\tau_{l}$ le taux de TVA sur le marché $l, Q_{f k l}$ la quantité de biens $k$ vendue par l'entreprise $f$ sur le marché $l, \widetilde{P}_{f k l}(Q)$ la fonction de demande inverse pour le bien $k$ sur le marché $l$ et $C_{f k}(\cdot)$ le coût variable de production. Le prix optimal s'exprime comme le taux de marge optimal pris sur le coût marginal de production, $M C_{f k}$, soit :

$$
\frac{\widetilde{P}_{f k l}}{1+\tau_{l}}\left(1-\frac{1}{\varepsilon_{f k l}}\right)=M C_{f k}
$$

où $M C_{f k} \equiv \frac{\partial C_{f k}}{\partial Q_{f k l}}$ et $\varepsilon_{f k l} \equiv \frac{\partial \widetilde{P}_{f k l}}{\partial Q_{f k l}} \frac{Q_{f k l}}{\widetilde{P}_{f k l}}$ l'élasticité-prix de la demande pour le bien $k$ produit par la firme $f$ et vendu sur le marché $l$, que nous supposons strictement supérieure à 1 .

Pour simplifier les écritures, on indicera par $i \equiv\{f, k, l\}$ un bien $k$ produit par une firme $f$ et vendu sur le marché $l$. Ainsi $P_{i} \equiv \frac{\widetilde{P}_{i}}{1+\tau_{l}}$ correspond au prix optimal net de la TVA. On note $\mu_{i} \equiv\left(\frac{\varepsilon_{i}}{\varepsilon_{i}-1}\right)$ le taux de marge spécifique à la firme pour le produit et le marché considéré. Le prix optimal se ré-écrit dès lors sous la décomposition suivante :

$$
\log P_{i}=\log \mu_{i}+\log M C_{f k} .
$$

À la différence du taux de marge, le coût marginal, $M C_{f k}$, est spécifique à la firme et au type de bien produit mais ne dépend pas de la destination retenue. Ceci signifie que les coûts de transport et de distribution sont exclus de la fonction de coût de l'exportateur. De fait, notre modèle décrit le prix optimal d'un exportateur à la frontière de son propre pays, c'est-à-dire avant que

\footnotetext{
${ }^{2}$ Par exemple, Berman et al. (2009) ou Gopinath \& Rigobon (2008) trouvent des pass-through moyens aux alentours ou en dessous de $20 \%$.
} 
les droits de douane, les coûts de transport et de distribution ne soient facturés. Cependant notre spécification n'exclut pas le fait qu'un bien $k$ produit par une société $f$ soit vendu à un prix différent selon la destination finale $l$. Ces écarts à la loi du prix unique proviennent ici fondamentalement de différences nationales dans l'élasticité de la demande adressée à cette société pour ce produit. Ces différences d'élasticités de demande peuvent indirectement provenir de différences dans les coûts de transport ou de distribution car un bien coûteux à distribuer dans un pays aura une demande locale plus réduite, et il n'y a aucune raison de penser que l'élasticité de la demande soit constante. Cependant, il est important de noter qu' un choc de TVA affecte le prix du bien indépendamment de la source de son approvionnement. A la différence des apports de Corsetti \& Dedola (2005) et Berman et al. (2009) qui soulignent l'importance des coûts de distribution dans la compréhension du traditionnel coefficient de "pass-though", ces coûts ne semblent pas déterminants dans la transmission des chocs de TVA sur les prix des exportateurs.

Nous modélisons l'élasticité de la demande adressée à la firme comme une fonction générale, non spécifiée de l'élasticité de la demande spécifique au secteur, $\varepsilon_{k}$, de la part de marché de la firme $f$ sur le marché du bien $k$ dans le pays $l$ au sein des exportateurs français, $s_{i} \equiv P_{i} Q_{i} / \sum_{j \in(k, l)} P_{j} Q_{j}$ et un vecteur de caractéristiques propres à la firme $Z_{f}$ :

$$
\varepsilon_{i} \equiv \varepsilon\left(\varepsilon_{k}, s_{i}, Z_{f}\right)
$$

où, par normalisation, $\varepsilon\left(\varepsilon_{k}, 1, \bar{Z}_{f \in K}\right)=\varepsilon_{k}$, avec $\bar{Z}_{f \in k}$ moyenne des caractéristiques des firmes parmi les entreprises présentes sur le marché du bien $k$. Cette fonction d'élasticité perçue de la demande nous permet d'écrire la fonction de taux de marge optimal :

$$
\mu_{i}=\frac{\varepsilon\left(\varepsilon_{k}, s_{i}, Z_{f}\right)}{\varepsilon\left(\varepsilon_{k}, s_{i}, Z_{f}\right)-1} \equiv \mu\left(\varepsilon_{k}, s_{i}, Z_{f}\right)
$$

Cette spécification couvre plusieurs modèles théoriques de concurrence imparfaite en économie internationale ${ }^{3}$. Elle peut aussi être étendue à des stratégies de prix prenant en compte le niveau futur anticipé de la part de marché de la firme ${ }^{4}$.

\subsection{Choc de TVA et variations optimales des taux de marge}

Sous l'hypothèse de rendements d'échelle constants, le coût marginal n'est pas influencé par le taux de TVA du marché de destination $l$. La variation du taux de marge optimal se répercute ainsi directement dans le prix optimal net, sans aucune interférence du coût marginal :

$$
\sigma\left(P_{i}\right)=\sigma\left(\mu_{i}\right)=\frac{-\sigma_{\varepsilon_{i}}}{\varepsilon_{i}-1}
$$

\footnotetext{
${ }^{3}$ Voir par exemple Melitz \& Ottaviano (2008) ou Atkeson \& Burstein (2008).

${ }^{4}$ Voir par exemple Froot \& Klemperer (1989).
} 
Où $\sigma_{\varepsilon_{i}}=\frac{d \varepsilon_{i}}{d P_{i}} \frac{P_{i}}{\varepsilon_{i}}$ est l'élasticité prix de l'élasticité de la demande, $\sigma\left(\mu_{i}\right)$ l'élasticité du taux de marge au taux de TVA et $\sigma\left(P_{i}\right)=\frac{d P_{i}}{d\left(1+\tau_{l}\right)} \frac{\left(1+\tau_{l}\right)}{P_{i}}$ l'élasticité du prix net au taux de TVA. Cette équation peut être écrite sous la forme log-linéaire suivante :

$$
d \log P_{i}=\frac{-\sigma_{\varepsilon_{i}}}{\varepsilon_{i}-1} d \log \left(1+\tau_{l}\right)
$$

L'équation (3) indique que l'exportateur réduira son prix net à la suite d'une augmentation du taux de TVA dans le pays de destination lorsque l'élasticité-prix de la demande est une fonction croissante du prix en niveau. Au contraire, si l'élasticité-prix est constante $\left(\sigma_{\varepsilon_{i}}=0\right)$, il n'y aura aucun ajustement des prix producteurs. Dans ce dernier cas, une augmentation du taux de TVA se traduit par une augmentation strictement proportionnelle des prix TTC (sans changement du prix producteur) : le coût lié à l'augmentation de la TVA est entièrement supporté par les consommateurs. Comme le soulignent Anderson et al. (2001), un producteur peut très bien choisir de sur-réagir à la hausse ou à la baisse selon la valeur de l'élasticité de l'élasticité de la demande.

La littérature récente a souligné l'importance de la prise en compte de l'hétérogénéité des firmes dans la compréhension de nombreux faits stylisés en commerce international. Un choc de demande peut induire une modification des marges intensives, mais aussi des marges extensives du commerce, au sens où il est important de tenir compte des entrées et des sorties des entreprises sur un marché. Notre modèle prend en compte cette hétérogénéité de sorte que la réponse d'un producteur au choc spécifique de destination (ici, un choc de TVA) provient d'une modification de l'élasticité de la demande qui est spécifique à chaque produit du secteur $k$, mais aussi du niveau de sa part de marché.

\subsection{Stratégie Économétrique}

Supposons que le prix pour le produit noté $p_{i} \equiv \log P_{i}, i \equiv\{k, f, l\}$ soit observé aux dates successives $t=1, \ldots, T$. Une contrepartie empirique à l'équation (3) peut s'écrire sous la forme :

$$
\Delta p_{i t}=-\sigma_{\varepsilon_{i}}\left(\frac{1}{\varepsilon_{i}-1}\right) \Delta \log \left(1+\tau_{l t}\right)+\Delta e_{i t}
$$

où $\Delta e_{i t}$ représente les variations de la demande et des coûts marginaux qui ne sont pas reliés au choc de TVA. Ce terme peut être décomposé de la façon suivante :

$$
\Delta e_{i t}=\alpha_{i}+\theta_{t}+\gamma^{\prime} \Omega_{i t}+u_{i t}, \quad \mathrm{E}\left\{u_{i t} \mid \theta_{t}, \Omega_{i t}\right\}=0
$$

avec $\Omega_{i t}$ un ensemble de facteurs pouvant influencer la demande d'importation spécifique au bien $i$ c'est à dire, au marché de destination $l$, au produit $k$ et à la firme $f$. 
Nous supposons par ailleurs que le choc de TVA est exogène ${ }^{5}$, c'est à dire que :

$$
\mathrm{E}\left\{u_{i t} \mid \theta_{t}, \Omega_{i t}, \Delta \log \left(1+\tau_{l t}\right)\right\}=\mathrm{E}\left\{u_{i t} \mid \theta_{t}, \Omega_{i t}\right\}=0 \text {. }
$$

Finalement, nous supposons que l'élasticité de l'élasticité de la demande est homogène pour l'ensemble des biens $i \equiv\{f, k, l\}$ :

$$
\sigma_{\varepsilon_{i}}=\sigma \quad \forall i
$$

Cette dernière hypothèse n'entraîne pas nécessairement que la réaction des prix des producteurs soit identique d'une entreprise à l'autre, ni même en moyenne d'un secteur à l'autre. En effet, cette spécification tient compte de la réaction hétérogène des prix à la TVA qui est donnée par $\frac{-\sigma}{\varepsilon_{i}-1}$. De là, notre identification implique des réactions hétérogènes substantielles entre les secteurs.

Sous ces hypothèses, en notant $\Delta r_{i t} \equiv\left(\frac{1}{\varepsilon_{i}-1}\right) \Delta \log \left(1+\tau_{l t}\right)$, l'équation (4) peut finalement s'écrire de la façon suivante :

$$
\Delta p_{i t}=\alpha_{i}+\theta_{t}-\sigma \Delta r_{i t}+\gamma^{\prime} \Omega_{i t}+u_{i t}, \quad \mathrm{E}\left\{u_{i t} \mid \theta_{t}, \Delta r_{i t}, \Omega_{i t}\right\}=0
$$

Une difficulté empirique est que $\Delta r_{i t}$ n'est pas directement observable puisque l'élasticité $\varepsilon_{i}$ n'est pas directement disponible à ce niveau fin de classification des produits (NC8). Nous traitons ce problème en remplaçant $\varepsilon_{i}$ par les élasticités sectorielles/pays $\varepsilon_{s l}$ estimées par Broda \& Weinstein (2006) aun iveau HS3 dans chaque pays $l$. Ainsi, $\Delta r_{i t}$ est approximé par :

$$
\Delta \widetilde{r}_{s l t} \equiv\left(\frac{1}{\varepsilon_{s l}-1}\right) \Delta \log \left(1+\tau_{l t}\right),
$$

de telles sortes, que nous pouvons estimer l'équation suivante :

$$
\Delta p_{i t}=\alpha_{i}+\theta_{t}-\sigma \Delta \widetilde{r}_{s l t}+\gamma^{\prime} \Omega_{i t}+\widetilde{u}_{i t}, \quad \mathrm{E}\left\{\widetilde{u}_{i t} \mid \theta_{t}, \Delta \widetilde{r}_{s t}, \Omega_{i t}\right\}=0 .
$$

L'estimation de cette équation nous permet d'obtenir un estimateur de l'élasticité de l'élasticité de la demande, $\widehat{\sigma}$. Les coefficients de "pass-through" pourront dès lors être recalculés au niveau pays/secteur :

$$
\text { Pass-through }_{s l}=1-\frac{\widehat{\sigma}}{\varepsilon_{s l}-1} \text {. }
$$

\footnotetext{
${ }^{5}$ Cette hypothèse est justifiée par le fait que les modifications du taux de TVA sont des décisions politiques exogènes. Cependant cette hypothèse est erronée si les entreprises utilisent des technologies à rendements d'échelle croissants. En effet, un changement d'ensemble du niveau de la demande induite par un changement du taux de TVA modifiera alors l'échelle de la production et affectera donc le coût marginal de l'entreprise. Dans la mesure où la modification du taux de TVA n'a lieu que dans un seul pays de destination, qui ne représente généralement qu'une fraction limitée de la production totale d'une entreprise exportatrice, l'argument lié à l'existence de rendements d'échelle croissants n'apparaît cependant pas très contraignant.
} 
Une extension consiste à estimer l'équation (7) pour des sous-populations d'entreprises exportatrices, classées selon leur part de marché, $m s_{i}$, qui influence l'élasticité de la demande adressée à une entreprise. On obtient ainsi des coefficients de "pass-through" hétérogènes variant avec le secteur d'activité, $s$, le pays de destination, $l$, mais aussi le niveau de la part de marché des entreprises.

\section{DONNÉES}

L'ensemble de données fourni par l'administration douanière française contient les exportations annuelles par pays de destination et par type de produit pour chaque entreprise individuelle au cours de la période $1995-2005^{6}$, de façon quasiment exhaustive.

Chaque observation est reliée à une firme $f$ (code siren), un produit défini à un niveau de désagrégation très fin (niveau NC8) $k$, ainsi qu'à une destination d'exportation $l$ et une année $t$. Les valeurs unitaires sont calculées comme le rapport entre les valeurs et les volumes déclarés. Ces valeurs unitaires sont utilisées comme proxy des prix unitaires d'exportation franco à bord : $P_{f k l t} \equiv P_{i t}$.

Les taux de TVA sont issus de European-Commission (2009). On observe une modification des taux normaux de TVA à huit reprises sur la période 1995-2005. Seul un des changements correspond à une baisse (l'Irlande en 2001). Pour les taux réduits, notre échantillon couvre trois hausses.

Nous limitons la base de données aux exportations de biens de consommation finale, expédiées au sein des 11 pays fondateurs de la zone euro ${ }^{7}$. La sélection de cet échantillon nous permet d'annihiler différentes sources majeures de fluctuations des prix internationaux comme une forte volatilité des taux de change, ou des modifications des droits de douane, pour nous concentrer sur notre problématique fiscale. Nous contrôlons pour les effets liés à la conjoncture macroéconomique en introduisant des dummies temporelles, qui capturent les chocs communs à l'ensemble des États membres de la zone euro, ainsi que des variables macro-économiques spécifiques aux différents pays, telles que le taux de croissance du PIB par tête ou les taux d'inflation, variables toutes issues d'Eurostat ou de la Banque Mondiale (World Development indicators).

Les élasticités-prix sectorielles proviennent de Broda \& Weinstein (2006), avec des informations pour environ 170 secteurs selon la classification HS3. La distribution de ces élasticités

\footnotetext{
${ }^{6}$ Cette base de données a été notamment déjà utilisée par Eaton et al. (2004), M ?jean \& Schwellnus (2009), Berman et al. (2009). A l'instar de différents auteurs, et pour contourner les problèmes liés aux changements de seuils de déclaration qui ont eu lieu au cours de cette période, nous avons choisi de nous concentrer sur les entreprises qui déclarent exporter à l'interieur de l'Union Européenne des montants supérieurs à 100,000 euros annuels. Nous avons par ailleurs tenu compte des changements dans la nomenclature de classification des produits sur la période.

${ }^{7}$ Nous avons exclu la Belgique comme pays de destination à cause de la non disponibilité d'estimation d'élasticités de la demande, $\varepsilon_{s l}$, pour ce pays.
} 
pour les biens de consommation finale est caractérisée par une médiane de 3,29 et une moyenne de 4,76 .

Quelques statistiques descriptives pour notre échantillon d'exportateurs français révèlent la grande hétérogénité des entreprises dans notre échantillon. Au sein d'un pays de destination spécifique, l'exportateur français médian vend 24 types de marchandises différents, avec un nombre de marchandises vendues par pays pouvant aller de 1 pour les plus petits exportateurs à 1762 pour les exportateurs les plus diversifiés.

\section{Resultats}

L'équation (7) est estimée en utilisant des effets fixes et des dummys temporelles. Le vecteur de variables de contrôle $\Omega_{i t}$ intègre en particulier la variable retardée $p_{i t-1}$ pour tenir compte de la viscosité des prix. La procédure d'Arellano-Bond est donc mise en oeuvre du fait du caractère auto-régressif de l'équation à estimer. Les tests de significativité tiennent compte des potentielles hétéroscedasticité et auto-corrélation des résidus. Les résultats sont reportés dans le Tableau (1). La colonne (1) fournit l'estimation de référence. Dans la colonne (2), nous restreignons l'échantillon aux entreprises qui résistent au choc de TVA, au sens où elles sont encore présentes après le choc. La colonne (3) reporte des résultats sur le même sous-échantillon, en estimant la réaction des entreprises selon leur part de marché. Plus précisément, nous croisons notre variable de choc de TVA avec une indicatrice indiquant à quel quartile de la distribution des parts de marché l'entreprise appartient.

La première ligne du Tableau (1) montre qu'une hausse du taux de TVA d'un pays de destination induit une baisse des prix d'exportations vers ce pays. À la suite d'une hausse de TVA dans un pays de destination, des exportateurs français font face en moyenne à une hausse de l'élasticité de la demande sur ce marché et réagissent donc en réduisant leur taux de marge.

Pour calculer le coefficient de "pass-though", le coefficient estimé ( $\hat{\sigma})$ doit être rapproché des élasticités de demande selon l'équation (8). Nous obtenons ainsi un "pass-though" moyen de $1-(1.824 / 3.76) \simeq 48 \%$ pour les biens de consommation finale. Cette estimation est plus élevée que les résultats récents reportés dans la littérature sur données individuelles, qui sont de l'ordre ou en dessous de $20 \%$, ce qui est conforme au fait que nous nous concentrons sur des chocs exogènes, symétriques, laissant le coût marginal constant. Il est en revanche plus surprenant d'observer que le coefficent de "pass-though" reste malgré tout très inférieur à l'unité, et qu'il n'est pas une spécificité des fluctuations des taux de change. Les colonnes (1) \& (2) montrent que les entreprises les plus résistantes ont un "pass-though" légèrement inférieur, avec un $\sigma$ de 1,989 plutôt que 1,824 .

Ces résultats peuvent être comparés avec ceux de Berman et al. (2009) qui montrent que les sociétés les plus productives ajustent leur taux de marge plus fortement que la moyenne. Cependant nos résultats soulignent l'importance d'autres caractéristiques dans la réaction du taux de marge optimal qui peuvent être non systématiquement liées à la productivité. En effet, si 
Tableau 1 : Estimation de l'équation de pass-through, (7)

(biens de consommation finale)

\begin{tabular}{|c|c|c|c|}
\hline & (1) & (2) & (3) \\
\hline VARIABLES & $\Delta p_{i t}$ & $\Delta p_{i t}$ & $\Delta p_{i t}$ \\
\hline$\overline{\mathrm{VAT}}_{l t}^{\varepsilon}$ & $\begin{array}{c}-1.824 * * * \\
(0.385)\end{array}$ & $\begin{array}{c}-1.989 * * * \\
(0.498)\end{array}$ & \\
\hline$\Delta \mathrm{VAT}_{l t}^{\varepsilon} \times Q_{4}^{s_{m}}$ & & & $\begin{array}{c}-1.934 * * * \\
(0.628)\end{array}$ \\
\hline$\Delta \mathrm{VAT}_{l t}^{\varepsilon} \times Q_{3}^{s_{m}}$ & & & $\begin{array}{l}-1.114 \\
(0.821)\end{array}$ \\
\hline$\Delta \mathrm{VAT}_{l t}^{\varepsilon} \times Q_{2}^{s_{m}}$ & & & $\begin{array}{l}-0.518 \\
(0.979)\end{array}$ \\
\hline$\Delta \mathrm{VAT}_{l t}^{\varepsilon} \times Q_{1}^{s_{m}}$ & & & $\begin{array}{c}-5.998 * * * \\
(2.194)\end{array}$ \\
\hline Number of id & 181092 & 134135 & 134135 \\
\hline
\end{tabular}

${ }^{a}$ Le détail des résultats pour les variables de contrôle est disponible auprès des auteurs. 
les firmes les plus productives sont aussi en général celles qui disposent des parts de marché les plus importantes, la relation n'est pas univoque. Une entreprise globalement très productive peut réagir différemment selon les marchés considérés, et plus précisément selon la part de marché dont elle dispose sur un marché. Cette part de marché peut provenir certes de sa productivité, mais aussi d'une plus ou moins grande connaissance des réseaux de distribution, de l'ancienneté de la firme sur le marché, ou de la structure concurrentielle.

Ainsi la colonne 3 du Tableau (1) montre que les entreprises ayant la plus grande part de marché $\left(Q_{4}^{s}\right)$ ajustent davantage leur taux de marge que les entreprises médianes. Mais on s'aperçoit aussi que les entreprises disposant des parts de marché les plus faibles réagissent de façon très importante pour préserver leur position sur le marché. Le coefficient de pass through n'est donc pas une fonction linéaire de la part de marché des entreprises.

\section{Conclusion}

L'utilisation de données individuelles sur les exportations françaises nous a permis de déterminer l'impact d'un choc de TVA dans un des pays de la zone euro sur le taux de marge des entreprises françaises. Nous montrons que les firmes françaises absorbent une part importante du choc dans leurs prix de production. Les caractéristiques du choc - exogène et sans incidence sur le coût marginal - nous permettent d'identifier que ces changements proviennent d'une modification des taux de marge, liée à une modification de l'élasticité-prix de la demande dans le pays de destination.

Le coefficient de "pass-though" moyen est estimé à 48\%. Ce chiffre est plus élevé que ceux généralement trouvés dans la littérature empirique sur données individuelles à la suite d'un choc de change, ce qui n'est pas surprenant en soi. Il est plus surprenant de trouver un "passthough" aussi incomplet pour un choc par nature symétrique pour l'ensemble des producteurs. Ce résultat est à notre sens une nouvelle preuve empirique validant les modèles théoriques où l'élasticité de la demande n'est pas supposée constante.

Une conséquence de nos résultats est que les prix à la consommation ne réagiront pas "un pour un" à une hausse du taux de TVA. Cependant, le mécanisme de compression de marge engendre une modification de la structure concurrentielle parmi les fournisseurs. Ce faisant, on peut craindre que les marchés deviennent plus concentrés avec des effets négatifs sur la disponibilité d'une vaste gamme de variétés à la suite d'une hausse de la TVA.

\section{RÉFÉRENCES}

Anderson, S., De Palma, A., \& Kreider, B. (2001). Tax incidence in differentiated product oligopoly. Journal of Public Economics, 81(2), 173-192.

Atkeson, A. \& Burstein, A. (2008). Pricing-to-market, trade costs, and international relative prices. American Economic Review, 98(5), 1998-2031. 
Berman, N., Martin, P., \& Mayer, T. (2009). How do different exporters react to exchange rate changes? Theory, empirics and aggregate implications. working paper 7493, CEPR.

Bouakez, H. \& Rebei, N. (2008). As the exchange rate pass-through really declined ? Evidence from Canada. Journal of International Economics, (pp. 245-67).

Broda, C. \& Weinstein, D. (2006). Globalization and the gains from variety. The Quarterly Journal of Economics, 121(2), 541-585.

Corsetti, G. \& Dedola, L. (2005). A macroeconomic model of international price discrimination. Journal of International Economics, 67, 129-155.

Eaton, J., Kortum, S., \& Kramarz, F. (2004). Dissecting trade : Firms, industries, and export destinations. American Economic Review, Papers and Proceedings, 93, 150-154.

European-Commission (2009). VAT Rates Applied in the Member States of the European Community. Technical report, Brussels.

Feenstra, R. (1989). Symmetric pass-through of tariffs and exchange rates under imperfect competition. Journal of International Economics, 27, 25-45.

Froot, K. \& Klemperer, P. (1989). Exchange rate pass-through when market share matters. American Economic Review, 79, 637-654.

Goldberg, L. \& Campa, J. (2008). The insensitivity of the cpi to exchange rates ; : Distribution margin, imported inputs and trade exposure. Review of Economics and Statistics.

Goldberg, P. \& Knetter, M. (1997). Goods prices and exchange rates : What have we learned ? Journal of Economic Literature, 35, 1243-72.

Gopinath, G. \& Rigobon, R. (2008). Sticky borders. Quarterly Journal of Economics, 123(2).

Knetter, M. (1989). Price discrimination by U.S. and German exporters. American Economic Review, 79, 198-210.

Knetter, M. (1993). An international comparison of pricing-to-market behaviour. American Economic Review, 83, 473-486.

Melitz, M. \& Ottaviano, G. (2008). Market size, trade, and productivity. Review of Economic Studies, 75, 295-316.

M ?jean, I. \& Schwellnus, C. (2009). Price convergence in the European union : Whithin firms or composition of firms. Journal of International Economics. 


\section{LIST OF WORKING PAPERS RELEASED BY CEPII}

An Exhaustive list is available on the website: IIwww.cepii.fr.

No

2010-29

2010-28

2010-27

2010-26

2010-25

2010-24

2010-23

2010-22

2010-21

2010-20 The Art of Exceptions:

Sensitive Products in the Doha Negotiations

2010-19 Measuring Intangible Capital Investment: an Application to the "French data"
Authors

M. Aleksynska \& Y. Algan

G. Gaulier, E. Milet \&

D. Mirza

J. Fouré, A. Bénassy-Quéré \& L. Fontagné

S. Jean \& C. Mitaritonna

V. Bouvatier, A. LopezVillavicencio \& V. Mignon

G. Capelle-Blancard

G. Gaulier

S. Zignago

F. McCann

X. Chojnicki

R. Magnani

C. Gouel

C. Mitaritonna

M.P Ramos

V. Delbecque L. Nayman

2010-18 Clustering the Winners: The French Policy of L. Fontagné, P. Koenig, F. Competitiveness Clusters $\quad$ Mayneris \& S. Poncet

V. Coudert \& M. Gex

G. Capelle-Blancard \& Y. Tadjeddine

G. Capelle-Blancard \& S. Monjon 
2010-13 Gold and Financial Assets: Are There Any Safe Havens in Bear Markets?

2010-12 European Export Performance

2010-11 The Effects of the Subprime Crisis on the Latin American Financial Markets: An Empirical Assessment

2010-10 Foreign Bank Presence and its Effect on Firm Entry and Exit in Transition Economies

2010-09 The Distorted Effect of Financial Development on International Trade Flows

2010-08 Exchange Rate Flexibility across Financial Crises

2010-07 Crises and the Collapse of World Trade: the Shift to Lower Quality

2010-06 The heterogeneous effect of international outsourcing on firm productivity

2010-05 Fiscal Expectations on the Stability and Growth Pact: Evidence from Survey Data

2010-04 Terrorism Networks and Trade: Does the Neighbor Hurt

2010-03 Wage Bargaining and the Boundaries of the Multinational Firm

2010-02 Estimation of Consistent Multi-Country FEERs

2010-01 The Elusive Impact of Investing Abroad for Japanese Parent Firms: Can Disaggregation According to FDI Motives Help

2009-39 The Effects at Home of Initiating Production Abroad: Evidence from Matched French Firms

2009-38 On Equilibrium Exchange Rates: Is Emerging Asia Different?

2009-37 Assessing Barriers to Trade in the Distribution and Telecom Sectors in Emerging Countries

2009-36 Les impacts économiques du changement climatique : enjeux de modélisation
V. Coudert \& H. Raymond

A. Cheptea, L. Fontagné \& S. Zignago

G. Dufrénot, V. Mignon \& A. Péguin-Feissolle

O. Havrylchyk

. Berthou

V. Coudert, C. Couharde \& V. Mignon

A. Berthou \& C. Emlinger F. McCann

M. Poplawski-Ribeiro \& J.C. Rüle

J. de Sousa, D. Mirza \& T. Verdier

M. Bas \& J. Carluccio

B. Carton \& K. Hervé

L. Hering, T. Inui \& S. Poncet

A. Hijzen, S. Jean \& T. Mayer

A. López-Villavicencio \& V. Mignon

L. Fontagné \& C. Mitaritonna

P. Besson \& N. Kousnetzoff 
2009-35 Trade, Foreign Inputs and Firms’ Decisions: Theory and

M. Bas Evidence

2009-34 Export Sophistication and Economic Performance: Evidence from Chinese Provinces

2009-33 Assessing the Sustainability of Credit Growth: The Case of Central and Eastern European Countries

2009-32 How do different exporters react to exchange rate changes? Theory, empirics and aggregate implications

2009-31 Spillovers from Multinationals to Heterogeneous Domestic Firms: Evidence from Hungary

J. Jarreau \& S. Poncet

V. Coudert \& C. Pouvelle

N. Berman, P. Martin \& Thierry Mayer

G. Békés, J. Kleinert \& F. Toubal

2009-30 Ethnic Networks, Information, and International Trade: Revisiting the Evidence

G. J. Felbermayr, B. Jung \& F. Toubal

2009-29 Financial Constraints in China: Firm-level Evidence

2009-28 The Crisis: Policy Lessons and Policy Challenges \& H. Vandenbussche

A. Bénassy-Quéré,

B. Coeuré, P. Jacquet

\&J. Pisani-Ferry

2009-27 Commerce et flux financiers internationaux : MIRAGE-D

A. Lemelin

2009-26 Oil Prices, Geography and Endogenous Regionalism: Too Much Ado about (Almost) Nothing

2009-25 EU15 Trade with Emerging Economies and Rentier States: Leveraging Geography

G. Gaulier, F. Lemoine \& D. Ünal

2009-24 Market Potential and Development

2009-23 Immigration, Income and Productivity of Host Countries: A Channel Accounting Approach

2009-22 A Picture of Tariff Protection Across the World in 2004 MAcMap-HS6, Version 2

T. Mayer

A. Mariya \& A. Tritah

H. Boumellassa, D. Laborde Debucquet \& C. Mitaritonna

2009-21 Spatial Price Discrimination in International Markets

J. Martin

2009-20 Is Russia Sick with the Dutch Disease

V. Dobrynskaya \& E. Turkisch

2009-19 Économies d'agglomération à l'exportation et difficulté d’accès aux marchés

P. Koenig, F. Mayneris \& S. Poncet

2009-18 Local Export Spillovers in France

P. Koenig, F. Mayneris \& S. Poncet

2009-17 Currency Misalignments and Growth: A New Look using Nonlinear Panel Data Methods,

S. Béreau,

A. López Villavicencio \& V. Mignon 
2009-16 Trade Impact of European Measures on GMOs

Condemned by the WTO Panel

2009-15 Economic Crisis and Global Supply Chains

2009-14 Quality Sorting and Trade: Firm-level Evidence for French Wine

2009-13 New Evidence on the Effectiveness of Europe's Fiscal Restrictions

2009-12 Remittances, Capital Flows and Financial Development during the Mass Migration Period, 1870-1913

2009-11 Evolution of EU and its Member States'Competitiveness in International Trade

2009-10 Exchange-Rate Misalignments in Duopoly: The Case of Airbus and Boeing

2009-09 Market Positioning of Varieties in World Trade: Is Latin America Losing out on Asia?

2009-08 The Dollar in the Turmoil

2009-07 Term of Trade Shocks in a Monetary Union: An Application to West-Africa

2009-06 Macroeconomic Consequences of Global Endogenous Migration: A General Equilibrium Analysis

2009-05 Équivalence entre taxation et permis d'émission échangeables

2009-04 The Trade-Growth Nexus in the Developing Countries: a Quantile Regression Approach

2009-03 Price Convergence in the European Union: within Firms or Composition of Firms?

2009-02 Productivité du travail : les divergences entre pays développés sont-elles durables?

2009-01 From Various Degrees of Trade to Various Degrees of Financial Integration: What Do Interest Rates Have to Say
A. C. Disdier \& L. Fontagné

A. Bénassy-Quéré, Y. Decreux, L. Fontagné \& D. Khoudour-Casteras

M. Crozet, K. Head \& T. Mayer

M. Poplawski Ribeiro

R. Esteves

\& D. Khoudour-Castéras

L. Curran \& S. Zignago

A. Bénassy-Quéré, L. Fontagné \& H. Raff

N. Mulder, R. Paillacar \& S. Zignago

A Bénassy-Quéré, S. Béreau \& V. Mignon

L. Batté, A. Bénassy-Quéré, B. Carton \& G. Dufrénot

V. Borgy, X. Chojnicki, G. Le Garrec \& C. Schwellnus P. Villa

G. Dufrénot, V. Mignon \& C. Tsangarides

I. Méjean \& C. Schwellnus

C. Bosquet \& M. Fouquin

A. Bachellerie, J. Héricourt \& V. Mignon 
Organisme public d'étude et de recherche en économie internationale, le CEPII est placé auprès du Centre d'Analyse Stratégique. Son programme de travail est fixé par un conseil composé de responsables de l'administration et de personnalités issues des entreprises, des organisations syndicales et de l’Université.

Les documents de travail du CEPII mettent à disposition du public professionnel des travaux effectués au CEPII, dans leur phase d'élaboration et de discussion avant publication définitive. Les documents de travail sont publiés sous la responsabilité de la direction du CEPII et n'engagent ni le conseil du Centre, ni le Centre d'Analyse Stratégique. Les opinions qui y sont exprimées sont celles des auteurs.

Les documents de travail du CEPII sont disponibles sur le site : http//www.cepii.fr.

CEPII

113, RUE DE GRENELLE - 75700 PARIS SP 07

TÉL. 0153685500

WWW.CEPII.FR

ISSN : 1293-2574 